\title{
The evaluation of bio-mechanical properties of four different skull implants by finite element methods.
}

\author{
Zhigang Wan ${ }^{1 \#, ~ C h i w e n ~ H u a n g ~}{ }^{2 \#}$, Yunqing $\mathrm{Li}^{3}$, Caihong Wan ${ }^{4}$, Rongde Zhong,6\#* \\ ${ }^{1}$ Department of Neurology, the Second Affiliated Hospital of Nanchang University, Nanchang, PR China \\ ${ }^{2}$ Department of Neurosurgery, Ganzhou People's Hospital, Ganzhou, PR China \\ ${ }^{3}$ Department of Gastroenterology, Shanghai Tenth People's Hospital, Tongji University, Shanghai, PR China \\ ${ }^{4}$ School of Basic Medical Sciences, Capital Medical University, Beijing, PR China \\ ${ }^{5}$ Medical College of Nanchang University, Nanchang, PR China \\ ${ }^{6}$ Department of Neurosurgery, the Tenth People's Hospital of Shanghai, Shanghai, PR China \\ \#These authors have contributed equally to this work
}

\begin{abstract}
Cranioplasty helps to restore skull's integrity, stability the state of the intracranial structure, also to improve the aesthetic immensely and reduce the concerns caused by weakened of brain protection with decompressive craniectomy. By using stress-strain analyzing of the finite element methods, to analyse the shock resistance, ability of absorbing concussion and the stability after the implanting of four different skull implants, in the end to select the implant with the one with the best biological-mechanical properties combined with CT data of 2D imaging and computer-aided design technology to establish four implants: a. conventional 3D blanket titanium mesh; b. conventional wedge-shaped PEEK mesh; c. 3D blanket triangular parabolic titanium mesh with hole button; d. half wedge-shaped triangular parabolic PEEK mesh with hole button. To second by importing ansys-workbench 17.0 software, loading adding a 500N static force at the center of the implants area of $3.982 \mathrm{~cm}^{2}$ which simulate four different scenarios of the implants suffering a real impact of heavy weight or vehicles. Comparing stress distribution, the maximum stress, the strain distribution and the maximum deflection of four different implants and the corresponding defective skulls. Under 500N load force, both four implants and defective skulls didn't have breakdown or deformation, On the models a and $\mathrm{c}$ the stress concentrated at the temporal region of skull base. Pars orbitalis and the connecting areas by implants and defective skulls and the points of strength of the prosthesises. On the model $b$, the stress concentrated at the frontoparietal, the points of strength of the prosthesis, temporal region of skull base and titanium strip. The outcome of the model $d$ is similar to the case $b$, but without obvious stress concentration of triangular parabolic mesh with hole button. The $\sigma$ and $\varepsilon$ of implants a-d are respectively: $\mathbf{2 4 3 . 8 0 0}$ mpa; $21.002 \mathrm{mpa} ; 386.200 \mathrm{mpa} ; 2.731 \mathrm{mpa}$ and $0.1644 \mathrm{~mm} ; 0.0825 \mathrm{~mm}, 0.2782 \mathrm{~mm}, 0.0828 \mathrm{~mm}$. The $\sigma$ of defective skulls $\mathbf{c}$ is 6.039 mpa, its $\varepsilon$ is $0.0408 \mathbf{~ m m}$. The $\sigma$ and $\varepsilon$ of th defective skulls of $\mathbf{d}$ are accordingly 1.829 mpa and $0.0175 \mathrm{~mm}$. The mechanical properties of PEEK are superior to the titanium implant. It can take the better role of brain protection. $D$ of four prosthesis demonstrates the best ability in the biomechanics, followed by the $b$.
\end{abstract}

Keywords: Cranioplasty, Finite element methods, PEEK, Titanium, Bio-mechanics.

\section{Introduction}

Skull defect as a result of decompressive craniectomy which are used to treat severe head injury, massive cerebral infarction, cerebral hernia and brain tumor, needs further operation, the cranioplasty, if the economics allowed and there are no special circumstances (such as infection, intracranial hypertension and epilepsy), in order to improve intracranial homeostasis disorder caused by defective skull, external cerebral hernia, cognitive impairment, skull defect syndrome and restore the protective function of skull and its appearance [1-3]. In order to achieve a better aesthetics result and the biomechanical properties of implant post-operative and lower by- 
effects, with the help of IT technique, material science and bioengineering, all manufacturers, researchers and neurosurgeons have done significant research on material and form level and their achievements have been selling which for clinical implantation [4,5]. The clinical feedback following the implementation is related to many factors, such as biomechanical properties, in particular mechanical properties, are an important aspect. Traditional methods of mechanical research are not convenient to use in in vivo, while finite element methods can carry out effective mechanical analysis, visually, directly demonstrate the distribution of stress, stress concentration and strain of bones and implanted parts, it can also predict the failure of the implantation. Avoiding the shortcomings of the conventional mechanical studies which take longer time, unrepeatable, high costs $[5,6]$. To explore the mechanics changes, ability of absorbing concussion and the ability to skull protection of the implant and the bone after the operated patient under the external percussion, meanwhile to specify the shock resistance and stability of the implanted prosthesis [7]. By creating 3D finite element models of skull defect, this paper, when built and assembled four different implant models (conventional blanket 3D titanium mesh, conventional wedge-shaped PEEK mesh; 3D blanket triangular parabolic titanium mesh with hole button; half wedge-shaped triangular parabolic PEEK mesh with hole button). All models have been simplified and undergone finite element analysis. According to our knowledge, there has no many literatures regarding of the research on bio-mechanics of material and form level of implant with regard to simulation circumstance of trauma(post-operative). By using stress-strain analyzing of the finite element methods of the varied types of implants, to select relatively suitable implant for Cranioplasty and to provide authentic biomechanics evidence.

\section{Materials and Methods}

A volunteer, male, planned to cranioplasty underwent continuous spiral CT scanning (distance of screw pitch was $0.625 \mathrm{~mm}$, depth of stratum was $2 \mathrm{~mm}$, interval of layers was 2 $\mathrm{mm}$, collimation width was $20 \mathrm{~mm}$, rotate time was $1 \mathrm{~s}$, matrix was $512 \times 512$ ) and 178 pieces of the images was obtained. The images would be saved in DICOM format, afterward, imported into 3D reconstruction software named MIMICS 17.0 (Belgium Materialse) for images threshold division and images segmentation (HU1250-3515) to obtain the defective skull MASK. And then "DRAW" and "ERASE" of the "EDIT" function keys was applied on the MASK in the software, thus the unrelated tissue and constructed defect of the mask can be removed layer by layer to further distinguish and separate the skull and to get more accurate data. Function keys ("3D CALULATION", "SMOOTH" and "WRARP") was also applied on the mask to generate pictures of clear, intuitive and reappeared the three-dimensional structure of skull defects. It was saved in STL format. Following this, it was imported into 3-MATIC software (Belgium Materialse) to draw a design of mesh according to the principle of axisymmetric mirror, to draw a design of lock bottom according to the clinical experience and the actual curvature of the bone [8]. All models need to be materialized by using the software Geomagic Studio 2013 (US Geomagic), and go through noise reduction processing, elimination of the abnormal parts in form, while repairing holes and chamfering corners, so that models with smooth geometric surface were produced. At the end it was importing the UG8.5 (Germany, Siemens PLM Software), the purpose of this is to assemble parts (Figure 1) and to convert the format. The converted format was exported in XT format.

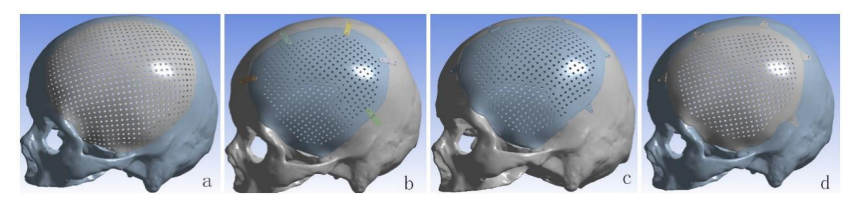

Figure 1. The positive morphology of 4 different implants already implanted.

2. The XT document was imported into the ansys-workbench 17.0 (US ANSYS). Considering the accuracy and few timeconsuming analyse and irregularity of skulls and prosthesises, we utilized a tetrahedral mesh and swept mesh segmentation, the number of nodes and units (Table 1). To make calculation easier, skulls and implants were considered as isotropic, homogeneous and continuous linear elastomer [9] and defined of material properties (Table 2). Although the behavior of the cancellous bone was taken into account during the simulation, it was not considered as a fracture criteria due to the superior mechanical strength properties of the cortical bone. The setting of the boundary condition was assumed at the bottom of skull, the impact on the implant by real heavy forces or vehicle was simulated by $500 \mathrm{~N}$ static force was vertically loaded on the prosthesises (the center implants area $3.982 \mathrm{~cm}^{2}$ ), then into the solution module, using model-static structural solver to carry out the stress-strain analysis.

\section{Result}

Under the condition of the same load, a finite elements analysis of stress-strain four implants was carried out. Figure 2 shows that the stress concentrated at the temporal region of skull base. Pars orbitalis and the connecting areas by implants and defective skulls and the points of strength of the prosthesises. On the model b, the stress concentrated at the frontoparietal, the points of strength of the prosthesis, temporal region of skull base and titanium strip. The outcome of the model $d$ is similar to the case $b$, but without obvious stress concentration of triangular parabolic mesh with hole button. Meanwhile, it was discovered that stress concentration of the implants of PEEK is stronger than the titanium prosthesisses which shows a gradual decreasing. The corresponding analysis data (Table 3) demonstrate that stress and strain of PEEK implants were both lower than titanium prosthesisses: a-d equivalent (von-mises) stress $(\sigma)$ and total deformation $(\varepsilon)$ were $243.800 \mathrm{mpa}, 21.002$ mpa, $386.2001 \mathrm{mpa}, 2.731 \mathrm{mpa}$ and $0.1644 \mathrm{~mm}, 0.0825 \mathrm{~mm}$, $0.2782 \mathrm{~mm}, 0.0828 \mathrm{~mm}$ respectively. The stress and strain of $\mathrm{c}$ is the maximum while the stress of $d$ is the minimum and its strain is nearly the same as b. Having implanted four implants, $\mathrm{c}$ has the maximum stress and strain ( $\sigma$ was 6.039 mpa, $\varepsilon$ was 
$0.0408 \mathrm{~mm}), \mathrm{b}$ is the minimum, $\mathrm{d}$ is slightly higher than $\mathrm{b}$. The $\sigma$ and $\varepsilon$ of the defective skull of d were $1.829 \mathrm{mpa}, 0.0175 \mathrm{~mm}$.

Table 1. Data of grid division of four implants.

\begin{tabular}{lll}
\hline Element type & No. of node & No. of elements \\
\hline A & 294043 & 1250455 \\
\hline B & 431914 & 257597 \\
\hline C & 200004 & 102530 \\
\hline D & 429874 & 257229 \\
\hline
\end{tabular}

Table 2. The characteristic and model parameters of implants and skull.

\begin{tabular}{llll}
\hline Materials & $\mathbf{E}(\mathbf{M P a})$ & $\mathbf{V}$ & $\mathbf{P}\left(\mathbf{g} / \mathbf{c m}^{3}\right)$ \\
\hline PEEK & 4000 & 0.4 & 1.3 \\
\hline Skull & 4000 & 0.3 & 1.45 \\
\hline titanium & 110300 & 0.33 & 4.5 \\
\hline
\end{tabular}
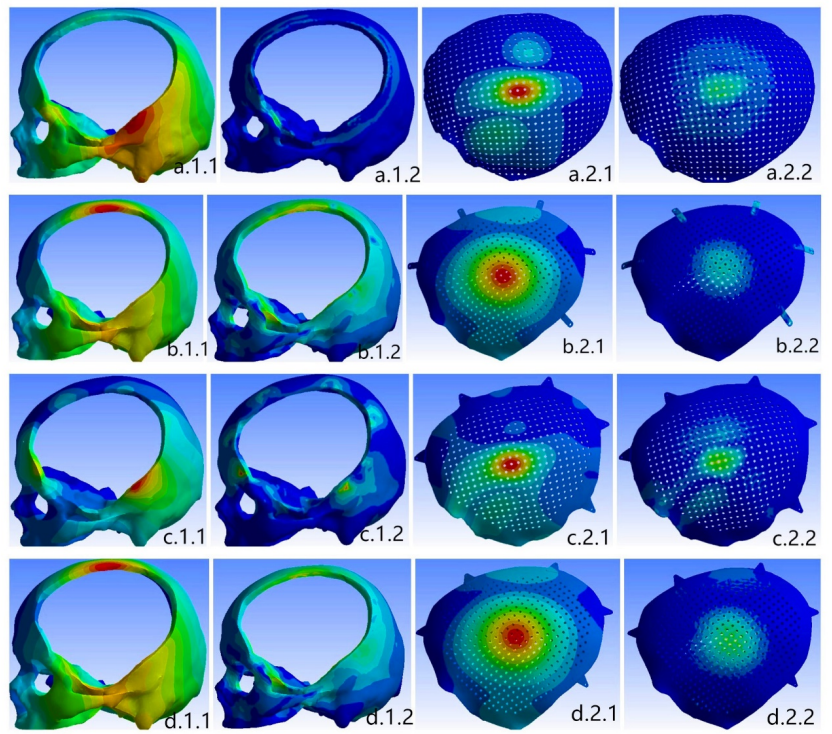

Figure 2. Results of cloud maps of stress-strain analysis of four implants and defective skulls. $A, b, c, d$ represent four type implants and defective skulls for Cranioplasty, $x .1 . x$ are the defective skulls, $x$. $2 . x$ are implants with difformity, x.x.1 assumed cloud map of stress, x.x.2 assumed cloud map of strain, $x$ can be $a, b, c, d, 1.2$. The red and yellow demonstrate the stress concentrated areas.

Table 3. The numerical results of stress and strain analysis of four implants and the defective skulls, equivalent (von-mises) stress $(\sigma)$ and total deformation $(\varepsilon)$.

\begin{tabular}{llll}
\hline \multicolumn{2}{l}{ Implants type } & Defective skull & implants \\
\hline $\mathrm{A}$ & $\sigma(\mathrm{mpa})$ & 5.424 & 243.8 \\
\cline { 2 - 4 } & $\varepsilon(\mathrm{mm})$ & 0.0142 & 0.1644 \\
\hline B & $\sigma(\mathrm{mpa})$ & 1.645 & 21.002 \\
\cline { 2 - 4 } & $\varepsilon(\mathrm{mm})$ & 0.0179 & 0.0825 \\
\hline
\end{tabular}

\begin{tabular}{llll}
\hline $\mathrm{C}$ & $\sigma(\mathrm{mpa})$ & 6.039 & 386.2 \\
\cline { 2 - 4 } & $\varepsilon(\mathrm{mm})$ & 0.0408 & 0.2782 \\
\hline $\mathrm{D}$ & $\sigma(\mathrm{mpa})$ & 1.829 & 12.731 \\
\cline { 2 - 4 } & $\varepsilon(\mathrm{mm})$ & 0.0175 & 0.0828 \\
\hline
\end{tabular}

\section{Discussion}

Cranioplasty can not only restore the integrity, the continuity of skull and the previous appearance, but also stabilize the intracranial pressure and create a intracranial stable state that facilitates the metabolism of brain tissues, restores the function of cranial nerves, re-establishes the brain protection and reduces the adverse consequences caused by the defect $[10,11]$. One of the key issues of cranioplasty are the material and shapes $[12,13]$. With the continuing development of materials science and the medical technique, all kinds of prosthesises keep emerging. Currently the widely used repairing material is titanium alloy and PEEK. As we know that properties of titanium alloy are light, easy to process and possess high biocompatibility. It has been the first choice for cranioplasty. PEEK, the so-called the pyramid of the resins industry, is one of the special engineering resins of high quality. It is used in the medical field enjoys the reputation of "the best long-term material for implanting". It possesses the qualities being heatresistant, radiation-resistant, abrasion-resistant and fatigueresistant, low-deformation, stable chemical performance, none genotoxic, none cytotoxicity and sensitization [14,15]. Its biocompatibility is excellent in long term. Eschenbach et al. pointed out that PEEK possesses the properties of being highly creep-resistant that it can take a great stress during its usage period, which won't stretch much in a long time [16]. According to the development reports of 2001 and 2012 from the resins industry, medical field consumes 10 percent of PEEK and it is mainly used in the operation instruments, prosthesises, bone nails, bone panels and so on [17,18]. Before PEEK has been applied for cranioplasty, it has been used as spine interbody fusion cage or Hip replacement device. Comparing with titanium alloy, PEEK has the following advantages: 1. PEEK possesses the similar elastic modulus of cortical bone (PEEK, E=3 4 GPa; cortex of bone, $\mathrm{E}=4 \sim 18$ $\mathrm{MPa}$; titanium, $\mathrm{E}=110 \mathrm{GPa})$, this means that $\mathrm{PEEK}$ is easier than titanium to osseointegration, so the failure rate of cranioplasty would be lower. Although PEEK and titanium both are of rigid solid, but PEEK possesses better flexibility, is able to adjust the deformation under the condition of homotaxial being stretched and compressed. The density of PEEK and titanium are $1.3 \mathrm{~g} / \mathrm{cm}^{3}, 4.5 \mathrm{~g} / \mathrm{cm}^{3}$, respectively. Obviously PEEK can provide patients better comfort after they have undergone large craniotomy. 2. It is radio-transparent and compatible with MRI. It will not produce artifacts on the CT and $\mathrm{X}$ ray as titanium alloy cause by ray attenuation so that post-operative re-examine and radiotherapy and other clinical treatments will not suffer [19]. 3. PEEK doesn't change because heat for low thermal conductivity, can perform better cerebral protection under the condition when the external temperature changes radically. But titanium alloy has highly 
yield strength and ultimate strength which makes it have a higher fracture mechanics, titanium alloy and PEEK loading above $45.8 \mathrm{kn}$ and $24.2 \mathrm{k}$ respectively will lead to fracture. During the experiment of mechanics of machinery by Yara and Bernd and in the study of stress-strain analysis of finite element methods by Alexander, finding both titanium implants and PEEK implants can take more force than skull (skull's breaking power 5.29 6.16 kn). But it has to emphasize that under the heavy loading, PEEK can relief and absorb the force of impact evenly by bending and transformation itself while titanium doesn't change accordingly and spreads the stress to the edge of bone which causes the breakdown of bone at edge. In other words, although titanium has higher breaking strength, but PEEK can protect brain better [4,7,20]. Steven and Ivan point out that PEEK has a lower bioactivity than titanium [14]. Yet Charles et al. discovered that fiber tissues and blood vessels, while they were doing test of the bio-compatibility, climbing along the PEEK fusion cage [20,21]. Brennan et al. have also achieved the same results during their experiment on cell-culture on Porous PEEK nets: cells proliferating, bone growing and vascularization. PEEK implants coated with hydroxyapatite, carbon fiber and other materials, can not only increase the mechanical rigidity, but also the bioactivity of PEEK [22]. As for the result after implanting, Ady then described that the complication and failure of the PEEK group and the titanium group are respectively $25.0 \%$ to $27.8 \%$ and $12.5 \%$ to $25 \%$ [23]. In $2009 \mathrm{Kim}$ et al. reported four cases of cranioplasty, followed-up post-operative in 16 to 20 months, resulting there is no infection, exposure, shifting, furthermore the appearance and the neurological function have been improved [24]. This study used PEEK and Titanium as material to design four kinds of prosthesises (Figures 1a-1d). The implants $\mathrm{a}$ and $\mathrm{b}$ are both widely used as cranioplasty' implants in clinical. The thickness of a was $0.8 \mathrm{~mm}$, size was $11.5 \times 13.5 \mathrm{~mm}, 1 \mathrm{~cm}$ beyond the edge of calvarial defect regions, diameter of the mesh was $2 \mathrm{~mm}$. Self-tapping screws of diameter $2 \mathrm{~mm}$ can be used to fix the implants on the skull surface, the thickness of $b$ was close to skull $(3.96-7.98 \mathrm{~mm})$, size was $10.5 \times 12.5 \mathrm{~cm}$. At regions (large area) of central distribution of porous holes, the diameter of the holes were 2.1 $\mathrm{mm}, 2.4 \mathrm{~mm}$ in distance, fixed by five piece of titanium connection strap with 2 holes at double ends $(0.5 \mathrm{~cm}$ in width, $0.6 \mathrm{~cm}$ in thickness, hole in diameter of $2 \mathrm{~mm}$ ) and 10 selftapping screws. Disadvantage of a after implanted are easy to nail pulling, titanium wires exposed, loose implants, the location of screw points are unregulated and rely on the experience to decide where besides, if the products are manufacture by dry pressing, it can happen that they won't fit with bone defect regions which lead to uniform pressure, needs to be re-shape during the operation. The stress of $b$ implant was not even, concentrating on the titanium strip, and, arranging for titanium strips relies on experience. To solve the problems above, we proposed to design the $\mathrm{c}$ and $\mathrm{d}$ prosthesises. Based on the implants of $\mathrm{a}$ and $\mathrm{b}$ of mesh hole button like para-curved triangle was added, mesh and hole button integrated, distributing and numbers of the hole button were decided through the finite element analysis again and again. During our study many interference factors had to be eliminated, distributing and numbers of the hole buttons were unified. The thickness of hole buttons were $1 \mathrm{~mm}$, the size were $21.6 \mathrm{~mm}$, diameter of the holes $2 \mathrm{~mm}$. The purpose of hole buttons were designed to cut operative time, clarify how to distribute screws by mechanical principles. $2 \mathrm{D}$ imaging of $\mathrm{CT}$ data and computer-aided design technique all were used in design four implants, so as to ensure that implants were connected on defect calvarium edge more accuracy, post-operative appearance more beautiful and be watched in 1:1 3D anatomical model and surgery simulation before cranioplasty. Considering with need of CPU, calculation time, and the project's goal is to analyse implants, titanium nails can be ignored.

Finite element methods are an analysis which disperses continuum into aggregations of a number of limited sized units, to solve the problems of aggregations mechanics. It has the traits of high efficacy, accuracy, simplicity and convenience, therefore is widely used in clinical to study complex mechanics [5]. It can load many loadings on models under different conditions, revising various test parameters, obtaining mechanics results of models under compression, bending, twisting and other loadings, more important is that it can provide the information about internal force of models by outported the stress nephogram and stress ribbon image and strain nephogram and strain ribbon image, one can be very intuitive understand how the stress concentration and damaged state of the implants models and the defective skull models, corresponding stress and strain values describe the mechanical strength of the implant and the stability after implantation, predicting of the result of operation, meanwhile the analysis results provides a guide for re-design of implants with regard to shapes and structures, while the conventional mechanical experiment in vitro is unable to do so [25]. Yara et al. had compared the results of standard machine impact test and finite element analysis, it haven't found obvious statistical difference, showing that finite element methods is reliable [26]. Our study applied $500 \mathrm{~N}$ static force vertically on the implants central zone of $3.982 \mathrm{~cm}^{2}$, to simulate the scenario of heavy force or vehicle impact on four prosthesis. A more suitable prosthesis can be decided by the results of the above.

Under $500 \mathrm{~N}$ loading four implants and defective Kulls didn't have break or deformation. The results of experiments shown that peek implant whether the shock resistance or the impact energy absorption ability were superior than titanium implants, providing better post-operative stability of implants and brain protection. The reason was that the value of stress and strain of PEEK implants and defective skulls are all lower than the ones of the comparable titanium models. Besides it's stress on implants are distributed in larger area and decrease continuously and gradually. The stress of defective skull concentrated only at frontoparietal and temporal region of skull base where skull were thick or cover with musculi temporalis. Stress concentration and stress value reflect the shock resistance of implants in certain, while the critical strain reflects the stability after the implanting, stress concentration and deflection predict the ability of absorb impact energy and brain protection. After deformation, in particular deformation 
is vertically, the pressure of brain must be increase and will trigger the cerebral contusion which is adverse for the neurological function to recover [7,27]. The stress of the model $\mathrm{b}$ focuses on the titanium strip (no obvious stress and strain concentration on the implant d), $\sigma 21.002 \mathrm{mpa}, \varepsilon 0.01502 \mathrm{~mm}$, easy to lead to titanium fracture. Therefore the model $\mathrm{d}$ is better than the $b$ in the bio-mechanics. The possible reason might be that the triangle para-curved lock button could absorb the shock energies in the homogenized way to a considerable degree. The model $\mathrm{c}$ has the weakest bio-mechanical properties, then is the model a. The reason lies in the thickness of the implants. The thickness of the titanium implant was 0.8 $\mathrm{cm}$, the ones of PEEK implant was skulls close to 3.96-7.98 $\mathrm{mm}$, the models a superior than c because coverage areas of implant a was larger, so that impact force can be easily distributed. Through the models $\mathrm{b}$ and $\mathrm{d}$ both shown concentration stress onrontoparietal, but the bone at this area is thicker and can resist a stronger impact. Taking all factors into account, the model $\mathrm{d}$ has the best bio-mechanical properties, more suitable for cranioplasty.

However there were some limitations to our research, such as mesh algorithm, the setting of load and boundary condition remain hypothetic, cannot fully reflect the actual situation of the clinic. The finite element analysis in vitro in this tests were simulation of post-operative bio-mechanical situation, that is to say, it is the bio-mechanical situation in an instant single force (500 N vertical impact force on the implants central area of $3.982 \mathrm{~cm}^{2}$ ), while the implanted parts in vivo is a long process, effected by numerous factors and trauma degree can be varied. Therefore four implants need further follow-up research when they are implanted.

\section{Conclusion}

Although the implant $\mathrm{d}$ is an improved b. although this research still had some defects, it can be no doubt about the reliability and value of the finite element analysis and the excellent mechanical ability of implant $d$ cannot be denied, further research needs to be carried on. The model after computer aided design, not only ensure the curvature and fitted greatly into defective skull after implantation for patients but also with satisfying appearance, then stress-strain analysis were carried out by finite element method, our goals were: 1 . Observation and prediction mechanical change after cranioplasty will perform under an impact of external forces, implants' ability to absorb impact force and of protection, clarify the shock resistance and the stability of implants after implanted; 2. Guiding for designs and improvement of the shapes of implants. Obtained implants (model d) were to ensure not only a high quality of appearance and long-term mechanical stability after implanted which can reduce the postoperative adverse effect, providing a relieved, optimistic, satisfied post-operative life.

\section{Interests Conflicts}

All authors of the paper acknowledge that any interests conflict doesn't exist in this paper.

\section{Ethical Issue}

The test has been approved by the Ethical Committee of the Shanghai People's Hospital No 10. The volunteer have their consent for the collection of research plan and CT data.

\section{References}

1. Kolias AG, Kirkpatrick PJ, Hutchinson PJ. Decompressive craniectomy: past, present and future. Nat Rev Neurol 2013; 9: 405-415.

2. Andrabi SM, Sarmast AH, Kirmani AR, Bhat AR. Cranioplasty: Indications, procedures, and outcome-an institutional experience. Surg Neurol Int 2017; 8: 91.

3. Piazza M, Grady MS. Cranioplasty. Neurosurg Clin N Am 2017; 28: 257-265.

4. Lethaus B, Safi Y, Ter Laak-Poort M, Kloss-Brandstatter A, Banki F, Robbenmenke C. Cranioplasty with customized titanium and PEEK implants in a mechanical stress model. J Neurotrauma 2012; 29: 1077-1083.

5. Choi AH, Conway RC, Ben-Nissan B. Finite-element modeling and analysis in nanomedicine and dentistry. Nanomedicine (Lond) 2014; 9: 1681-1695.

6. Chen Y, Miao Y, Xu C, Zhang G, Lei T, Tan Y. Wound ballistics of the pig mandibular angle: a preliminary finite element analysis and experimental study. J Biomech 2010; 43: 1131-1137.

7. Tsouknidas A, Maropoulos S, Savvakis S, Michailidis N. FEM assisted evaluation of PMMA and Ti6Al4V as materials for cranioplasty resulting mechanical behaviour and the neurocranial protection. Biomed Mater Eng 2011; 21: 139-147.

8. Kung WM, Chen ST, Lin CH, Lu YM, Chen TH, Lin MS. Verifying three-dimensional skull model reconstruction using cranial index of symmetry. PLoS One 2013; 8: 264-267.

9. Daas M, Dubois G, Bonnet AS, Lipinski P, Rignon-Bret C. A complete finite element model of a mandibular implantretained over denture with two implants: comparison between rigid and resilient attachment configurations. Med Eng Phys 2008; 30: 218-225.

10. Mah JK, Kass RA. The impact of cranioplasty on cerebral blood flow and its correlation with clinical outcome in patients underwent decompressive craniectomy. Asian J Neurosurg 2016; 11: 15-21.

11. Hutchinson PJ, Kolias AG, Timofeev IS, Corteen EA, Czosnyka M, Timothy J. Trial of Decompressive Craniectomy for Traumatic Intracranial Hypertension. N Engl J Med 2016; 375: 1119-1130.

12. Neovius E, Engstrand T. Craniofacial reconstruction with bone and biomaterials: review over the last 11 years. J Plast Reconstr Aesthet Surg 2010; 63: 1615-1623.

13. Fazel A, Aalai S, Rismanchian M, Sadr-Eshkevari P. Micromotion and stress distribution of immediate loaded implants: a finite element analysis. Clin Implant Dent Relat Res 2009; 11: 267-271. 
14. Kurtz SM, Devine JN. PEEK biomaterials in trauma, orthopedic, and spinal implants. Biomaterials 2007; 28: 4845-4869.

15. Panayotov IV, Orti V, Cuisinier F, Yachouh J. Polyetheretherketone (PEEK) for medical applications. J Mater Sci Mater Med 2016; 27: 118.

16. Eschbach L. Nonresorbable polymers in bone surgery. Injury 2000; 31: 22-27.

17. Renaud M, Farkasdi S, Pons C, Panayotov I, CollartDutilleul PY, Taillades H. A new rat model for translational research in bone regeneration. Tissue Eng Part C Methods 2015.

18. Ghiacci G, Graiani G, Ravanetti F, Lumetti S, Manfredi E, Galli C. Over-inlay block graft and differential morphometry: a novel block graft model to study bone regeneration and host-to-graft interfaces in rats. J Periodontal Implant Sci 2016; 46: 220-233.

19. Li HM, Fouracre NMJG, Society IDAE. The effects on polyetheretherketone and polyethersulfone of electron and /spl gamma/ irradiation. IEEE Trans Dielect El In 1999; 6: 295-303.

20. Rivard CH, Rhalmi S, Coillard C. In vivo biocompatibility testing of peek polymer for a spinal implant system: a study in rabbits. J Biomed Mater Res 2002; 62: 488-498.

21. Wenz LM, Merritt K, Brown SA, Moet A, Steffee AD. In vitro biocompatibility of polyetheretherketone and polysulfone composites. J Biomed Mater Res 1990; 24: 207-215.

22. Torstrick FB, Evans NT, Stevens HY, Gall K, Guldberg RE. Do surface porosity and pore size influence mechanical properties and cellular response to PEEK? Clin Orthop Relat Res 2016; 474: 2373-2383.

23. Thien A, King NK, Ang BT, Wang E, Ng I. Comparison of polyetheretherketone and titanium cranioplasty after decompressive craniectomy. World Neurosurg 2015; 83: 176-180.

24. Kim MM, Boahene KD, Byrne PJ. Use of customized polyetheretherketone (PEEK) implants in the reconstruction of complex maxillofacial defects. Arch Facial Plast Surg 2009; 11: 53-57.

25. Zhao Y, Zhang S, Sun T, Wang D, Lian W, Tan J. Mechanical comparison between lengthened and short sacroiliac screws in sacral fracture fixation: a finite element analysis. Orthop Traumatol Surg Res 2013; 99: 601-606.

26. Lethaus B, Safi Y, ter Laak-Poort M, Kloss-Brandstatter A, Banki F, Robbenmenke C. Cranioplasty with customized titanium and PEEK implants in a mechanical stress model. J Neurotrauma 2012; 29: 1077-1083.

27. Beainy F, El AC, Abousleimane Y, Mapstone T, Beidas O, Workman M. Biomechanical effects of cranioplasty for defects using autogenous calvarial bone. J Craniofac Surg 2012; 23: 152-155.

\section{*Correspondence to}

Rongde Zhong

Department of Neurosurgery

The Tenth People's Hospital of Shanghai

PR China 